\title{
Transport through Quantum Dots: Analytic Results from Integrability
}

\author{
R. M. Konik ${ }^{1,3}$, H. Saleur ${ }^{2}$, and A. W. W. Ludwig ${ }^{3}$ \\ ${ }^{1}$ Department of Physics, University of Virginia, Charlottesville, VA 22903 \\ ${ }^{2}$ Department of Physics, University of Southern California, Los-Angeles, CA 90089-0484 \\ ${ }^{3}$ Department of Physics, UCSB, Santa Barbara, CA 93106
}

(November 2, 2018)

\begin{abstract}
Recent experiments have probed quantum dots through transport measurements in the regime where they are described by a two lead Anderson model. In this paper we develop a new method to analytically compute for the first time the corresponding transport properties. This is done by using the exact solvability of the Anderson Hamiltonian, together with a generalization of the LandauerBüttiker approach to integrable systems. The latter requires proper identification of scattering states, a complex and crucial step in our approach. In the Kondo regime, our results include the zero-field, finite temperature linear response conductance, as well as the zero-temperature, nonequilibrium conductance in an applied Zeeman field.
\end{abstract}

In recent years there have been a flurry of experimental studies of quantum dots [1]2]. In these experiments a single localized dot state interacts with connecting leads notwithstanding that a finite number of electrons sits on the dot. In a testament to advances in the miniaturization of solid state technology, the occupancy of this level can be controlled by a gate voltage, thereby controlling the current through this 'single-electron transistor'. For an odd number of electrons, the dot becomes, due to Kramers degeneracy, a nanoscale realization of the single-impurity Kondo system - one has in effect a 'tunable' Kondo model [1,2]. The observed split peak structure in the non-equilibrium conductance in an applied Zeeman field is a hallmark of the Kondo physics 迥迏.

The experimental probes of these systems are measurements of transport properties. The appropriate theoretical framework to describe electronic transport through the dot is the two-lead Anderson model (to distinguish it from the one-lead model appropriate for studying dilute impurities in a bulk metal). This model has been examined using a variety of techniques. In 画, the $T=0$ linear response conductance was studied both in and out of a magnetic field via the Friedel sum rule (although the occupancy of the dot necessary to apply the rule was computed only in perturbation theory). Using the NCA approximation and perturbation theory, [5, 6] studied quantum dots out-of-equilibrium. However such techniques have limitations: the NCA approximation does not work in the presence of a magnetic field while perturbation theory requires either the dot Coulomb repulsion or the dot-lead coupling to be small. Numerically, the zerofield, finite temperature linear response conductance has been computed in [7] using the numerical renormalization group (NRG). However the latent integrability of the (one-lead) Anderson model 10,11] has never been exploited to compute directly transport properties.

In this work we combine the integrability of the Anderson model with Landauer-Büttiker transport theory.
To do so one must first face the challenge of identifying scattering states in the context of integrability. In [8] the Bethe ansatz of the simpler one-lead Kondo model was employed to study the equilibrium impurity DOS, of which the non-equilibrium counterpart, in context, would yield the desired transport properties [5]. Neither the computation of the latter nor the scattering states in the two lead model were addressed in [8]. The identification of the scattering states is non-trivial. The correct scattering states will necessarily be electronic in nature (i.e. carry charge e and spin 1/2) and will be confined to a single lead. In contrast, the eigenstates of the exact solution affect a spin-charge separation (i.e. they are not electronic) and they are not confined to a single lead. One purpose of this letter is to outline how one can understand scattering states in terms of the eigenstates of the Bethe ansatz.

We are successful in this endeavour on two counts. Firstly, we are able to construct scattering states at the Fermi surface for a dot under arbitrary gate voltage and arbitrary magnetic field and so are able to offer for the first time a proof of the Friedel sum rule based upon integrability, alternative to that from Fermi liquid theory [9]. Secondly, provided the gate voltage is adjusted so that the dot sits at the symmetric point, we can compute scattering states away from the Fermi surface. Through a Landauer-Bütiker approach we are then able to compute the zero-field finite temperature linear response conductance (in agreement with numerical results of [7]), as well as the $T=0$ non-equilibrium magneto-conductance, exhibiting the observed peak splitting.

Model: To then begin, the two-lead Anderson model Hamiltonian is given in the continuum limit by

$$
\begin{gathered}
\mathcal{H}=\sum_{l \sigma} \int_{-\infty}^{\infty} d x\left\{-i c_{l \sigma}^{\dagger}(x) \partial_{x} c_{l \sigma}(x)+V_{l} \delta(x)\left[c_{l \sigma}^{\dagger}(x) d_{\sigma}\right.\right. \\
\left.\left.+d_{\sigma}^{\dagger} c_{l \sigma}(x)\right]\right\}+\epsilon_{d} \sum_{\sigma} n_{\sigma}+U n_{\uparrow} n_{\downarrow},
\end{gathered}
$$

where $n_{\sigma}=d_{\sigma}^{\dagger} d_{\sigma}$. The $c_{l}$ 's are the lead electrons while 
the $d$ 's are the dot electrons. Here $\sum_{l}$ is a sum over the two leads $(l=1,2)$. Our formalism allows for the possibility that the hopping matrix element, $V_{l}$, differs between the leads (as is typical in any experimental realization). For simplicity, in our presented results we assume $V_{1}=V_{2}$. Rather than treating the leads as halflines with both left and right moving fermions, we represent the leads as 'unfolded' with fermions that are solely right-moving. Fermions in either lead that are incident upon the dot are considered to lie in the region, $x<0$, while those traveling away from the dot in either lead are found at $x>0$. We represent this in Figure 1 by drawing the leads as elongated arcs.

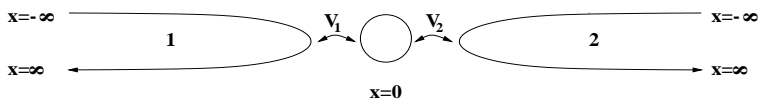

FIG. 1. A sketch of two leads attached to a quantum dot.

It will be advantageous to reformulate this problem as a one-lead Anderson Hamiltonian. To do so, we introduce even/odd electrons: $c_{e / o}=\left(V_{1} c_{1} \pm V_{2} c_{2}\right) / \sqrt{V_{1}^{2}+V_{2}^{2}}$. Recasting $\mathcal{H}$ in this new basis, the odd electron, $c_{o}$, decouples and we are left with

$$
\begin{aligned}
\mathcal{H}= & \sum_{\sigma} \int d x\left\{-i c_{e \sigma}^{\dagger}(x) \partial_{x} c_{e \sigma}(x)+\left(V_{1}^{2}+V_{2}^{2}\right)^{1 / 2} \delta(x)\right. \\
& \left.\times\left[c_{e \sigma}^{\dagger}(x) d_{\sigma}+d_{\sigma}^{\dagger} c_{e \sigma}(x)\right]\right\}+\epsilon_{d} \sum_{\sigma} n_{\sigma}+U n_{\uparrow} n_{\downarrow} .
\end{aligned}
$$

We have thus reduced the problem to that studied using Bethe ansatz in a series of papers by Kawakami and Okiji [10] and Filyov, Wiegmann and Tsvelick [1].

As such we summarize briefly the results of this work. Applying the Bethe ansatz to a system with $\mathrm{N}$ particles and total spin $2 S_{z}=(N-2 M)$ yields a set of quantization conditions describing a finite number of bare excitations in the system:

$$
\begin{aligned}
e^{i k_{j} L+i \delta\left(k_{j}\right)} & =\prod_{\alpha=1}^{M} \frac{g\left(k_{j}\right)-\lambda_{\alpha}+i / 2}{g\left(k_{j}\right)-\lambda_{\alpha}-i / 2} ; \\
\prod_{j=1}^{N} \frac{\lambda_{\alpha}-g\left(k_{j}\right)+i / 2}{\lambda_{\alpha}-g\left(k_{j}\right)-i / 2} & =-\prod_{\beta=1}^{M} \frac{\lambda_{\alpha}-\lambda_{\beta}+i}{\lambda_{\alpha}-\lambda_{\beta}-i}
\end{aligned}
$$

where $\delta(k)=-2 \tan ^{-1}\left(\Gamma /\left(k-\epsilon_{d}\right)\right), g(k)=\left(k-\epsilon_{d}-\right.$ $U / 2)^{2} / 2 U \Gamma$, and $\Gamma=\left(V_{1}^{2}+V_{2}^{2}\right)$.

When $\epsilon_{d}>-U / 2$ (the case $\epsilon_{d}<-U / 2$ can be handled through particle-hole transformations), the zero temperature ground state of the system is formed from $N-2 M$ real $k_{j}$ 's and $M$ real $\lambda_{\alpha}$ 's. Associated with each $\lambda_{\alpha}$ is a pair of complex k's, $k_{ \pm}^{\alpha}$, related to the $\lambda_{\alpha}$ via $g\left(k_{ \pm}^{\alpha}\right)=$ $g\left(x\left(\lambda_{\alpha}\right) \mp i y\left(\lambda_{\alpha}\right)\right), x(\lambda)=U / 2+\epsilon_{d}-\sqrt{U \Gamma}\left(\lambda+\left(\lambda^{2}+\right.\right.$ $\left.1 / 4)^{1 / 2}\right)^{1 / 2}$, and $y(\lambda)=\sqrt{U \Gamma}\left(-\lambda+\left(\lambda^{2}+1 / 4\right)^{1 / 2}\right)^{1 / 2}$. Roughly speaking, the real $k$ 's can be thought of as charge excitations and the $\lambda_{\alpha}$ 's as spin excitations coupled to charge excitations, i.e. bound states of electrons.
The finite temperature ground state is considerably more complicated, involving an infinite hierarchy of excitations as categorized in the 'string-hypothesis' 10,11.

Scattering States: Under this one-lead reformulation, we are still able to make contact with the scattering amplitudes of electronic excitations off the quantum dot. Let $T(\epsilon) / R(\epsilon)$ be the transmission/reflection amplitudes of electronic excitations of energy $\epsilon$ between leads in the original two lead picture. On the other hand, the even/odd excitations will scatter off the dot with some pure phase, $\delta_{e}(\epsilon) / \delta_{o}(\epsilon)$, where in particular $\delta_{o}(\epsilon)=0$. The two sets of amplitudes are related straightforwardly:

$$
\begin{aligned}
& e^{i \delta_{e}(\epsilon)}=R(\epsilon)+T(\epsilon) ; \\
& e^{i \delta_{o}(\epsilon)}=1=R(\epsilon)-T(\epsilon) .
\end{aligned}
$$

We will exploit extensively the fact that the determination of the phase, $\delta_{e}$, gives $T$ and $R$ in the original problem.

To determine $\delta_{e}(\epsilon)$, we employ an energetics argument of the sort used by N. Andrei in the computation of the $T=0$ magnetoresistance of impurities in a bulk metal 13]. Imagine adding an electron to the system. Through periodic boundary conditions, its momentum is quantized, $p=2 \pi n / L$. If the dot was absent, the quantization condition would be determined solely by the conditions in the bulk of the system and we would write, $p_{\text {bulk }}=2 \pi n / L$. Upon including the dot, this bulk momentum is shifted by a term scaling as $1 / L$. The quantization condition is then rewritten as

$$
p=2 \pi n / L=p_{\text {bulk }}+\delta_{e}(\epsilon) / L,
$$

where $L$ is the system's length. The coefficient of the $1 / L$ term is identified with the scattering phase of the electron off the dot.

In order to determine the scattering phase of an electron (as opposed to a spin or charge excitation), we must must specify how to glue together a spin and a charge excitation to form the electron. The situation is analogous to adding a single particle excitation in the attractive Hubbard model. Adding a single spin $\uparrow$ electron to the system demands that we add a real $k$ (charge) excitation. But at the same time we create a hole at some $\lambda$ in the spin distribution. The number of the available slots in the spin distribution is determined by the number of electrons in the system. Adding an electron to the system thus opens up an additional slot in the $\lambda$ distribution. The electron scattering phase off the impurity is then the difference of the right-moving k-impurity momentum, $p_{\text {imp }}(k)$, and the left-moving $\lambda$-hole impurity momentum $-p_{\text {imp }}(\lambda)$ :

$$
\delta_{e}^{\uparrow}=p_{\text {imp }}^{\uparrow}=p_{\text {imp }}(k)+p_{\text {imp }}(\lambda) .
$$

We now turn our attention to computing these impurity momenta. As part of this, we will relate the impurity momenta to the impurity density of states (which in turn, 
will allow us to prove the Friedel sum rule). We at the same time will compute the energy of the $k$ and $\lambda$ excitations in order to parameterize scattering in terms of energy.

In the continuum limit, these excitations are described by smooth densities, $\rho(k)$ for the real $k$ 's and $\sigma(\lambda)$ for the $\lambda$ 's. From (3), equations describing these densities can be derived in the standard fashion [11, 10]:

$$
\begin{gathered}
\rho(k)=\frac{1}{2 \pi}+\frac{\Delta(k)}{L}+g^{\prime}(k) \int_{Q}^{\tilde{Q}} d \lambda a_{1}(g(k)-\lambda) \sigma(\lambda) ; \\
\sigma(\lambda)=-\frac{x^{\prime}(\lambda)}{\pi}+\frac{\tilde{\Delta}(\lambda)}{L}-\int_{Q}^{\tilde{Q}} d \lambda^{\prime} a_{2}\left(\lambda^{\prime}-\lambda\right) \sigma\left(\lambda^{\prime}\right) \\
\quad-\int_{-D}^{B} d k a_{1}(\lambda-g(k)) \rho(k),
\end{gathered}
$$

where $L$ is the system size and $\Delta(k)=\partial_{k} \delta(k) / 2 \pi$, $\tilde{\Delta}(\lambda)=-\partial_{\lambda} \operatorname{Re} \delta(x(\lambda)+i y(\lambda)) / \pi$, and $a_{n}(x)=$ $2 n /\left(\pi\left(n^{2}+4 x^{2}\right)\right)$. $B$ and $Q$ mark out the 'Fermi-surfaces' of the $k$ and $\lambda$ distributions. A key observation to make of these equations is that one can divide the densities into bulk and impurity pieces via $\rho(k) \rightarrow \rho_{\text {bulk }}(k)+\rho_{\text {imp }}(k) / L$ and similarly for $\sigma(\lambda)$. The impurity densities of states contain all the information needed about degrees of freedom living on the quantum dot. For example the total numbers of spin $\uparrow$ and $\downarrow$ electrons living on the dot are $n_{d \uparrow}=\int_{Q}^{\infty} d \lambda \sigma_{\mathrm{imp}}(\lambda)+\int_{-\infty}^{B} d k \rho_{\mathrm{imp}}(k)$ and $n_{d \downarrow}=$ $\int_{Q}^{\infty} d \lambda \sigma_{\text {imp }}(\lambda)$.

The energies and momenta of these excitations can be derived through well known techniques 12. The energies are given by

$$
\begin{gathered}
\epsilon(k)=k-\frac{H}{2}-\int_{Q}^{\infty} d \lambda \epsilon(\lambda) a_{1}(\lambda-g(k)) ; \\
\epsilon(\lambda)=2 x(\lambda)-\int_{Q}^{\infty} \epsilon\left(\lambda^{\prime}\right) a_{2}\left(\lambda^{\prime}-\lambda\right) \\
\quad+\int_{-\infty}^{B} g^{\prime}(k) \epsilon(k) a_{1}(g(k)-\lambda) .
\end{gathered}
$$

The momenta are akin to the densities in that they divide into bulk and impurity pieces. The impurity momenta, the momenta relevant to scattering, are given by [12]

$$
\begin{aligned}
p_{\mathrm{imp}}(k)= & \delta(k)+\int_{Q}^{\tilde{Q}} d \lambda \sigma_{\mathrm{imp}}(\lambda) \theta_{1}(g(k)-\lambda) \\
p_{\mathrm{imp}}(\lambda)= & 2 \operatorname{Re} \delta(x(\lambda)+i y(\lambda))+\int_{Q}^{\tilde{Q}} d \lambda^{\prime} \sigma_{\mathrm{imp}}\left(\lambda^{\prime}\right) \theta_{2}\left(\lambda-\lambda^{\prime}\right) \\
& +\int_{-D}^{B} d k \rho_{\mathrm{imp}}(k) \theta_{1}(\lambda-g(k)),
\end{aligned}
$$

where $\theta_{n}(x)=2 \tan ^{-1}(x / n)-2 \pi$.

We now observe a relationship between the impurity DOS and the impurity momenta key to the actual computation of the scattering phases:

$$
\begin{aligned}
\partial_{k} p_{\text {imp }}(k) & =2 \pi \rho_{\text {imp }}(k) ; \\
\partial_{\lambda} p_{\text {imp }}(\lambda) & =-2 \pi \sigma_{\text {imp }}(\lambda) .
\end{aligned}
$$

The scattering phase for a spin $\uparrow$ excitation created from a $\mathrm{k}$-particle and $\lambda$-hole is then given to be

$$
\delta_{e}^{\uparrow}=2 \pi \int_{-\infty}^{k} d k \rho_{\mathrm{imp}}(k)+2 \pi \int_{\lambda}^{\infty} d \lambda^{\prime} \sigma_{\mathrm{imp}}\left(\lambda^{\prime}\right) .
$$

If $k$ and $\lambda$ are chosen to be at the Fermi surface, i.e. $k=$ $B$ and $\lambda=Q$, we prove the Friedel sum rule, i.e. $\delta_{e}^{\uparrow}=$ $2 \pi n_{d \uparrow}$. Through a particle-hole transformation, we can similarly characterize the scattering of spin $\downarrow$ excitations and so also prove the Friedel sum rule in this case.

To discuss scattering away from the Fermi surface, reconsider (10). Suppose we want to compute the scattering of a spin $\uparrow$ electron with energy, $\epsilon_{e l}$. If we choose the pair, $(k, \lambda)$, such that $\epsilon(\lambda)+\epsilon(k)=\epsilon_{e l}$, we will have, via (10), the scattering of an excitation with energy, $\epsilon_{e l}$. However we encounter a problem in that there is not a unique pair, $(k, \lambda)$, for a given energy. While we cannot solve the problem of choosing the correct pair (or combination of pairs) in general, we can make progress when we are in the Kondo regime of the Anderson model (i.e. $\left.U+2 e_{d} \sim 0\right)$. In this regime we expect the scattering phase to vary on the scale of the Kondo temperature, $T_{k}$. The electron scattering phase is determined by $\rho_{\text {imp }}$ and $\sigma_{\text {imp }}$, the two impurity densities. Of the two, only $\rho_{\text {imp }}$ varies on scales on the order of $T_{k}$ ( $\sigma_{\text {imp }}$ is controlled by the much larger scale, $\sqrt{U \Gamma}$, governing charge fluctuations). Thus in computing electronic scattering phases away from the Fermi surface at zero temperature, it is natural to keep $\lambda=Q$, its Fermi surface value, and vary $k$. Specifically, to describe an electron with energy, $\epsilon_{e l}$, we choose $(k, \lambda)$ such that $\lambda=Q$ and $k$ such that $\epsilon(k)=\epsilon_{e l}$. With this ansatz, we then have restricted the two dimensional phase space, $(\lambda, k)$, of potential excitations carrying the quantum numbers of an electron to an one dimensional subspace. We have further arguments that suggest this ansatz is exact at the symmetric point of the Anderson model [12].

Linear Response $(T>0)$ : We now try out this ansatz by examining a quantity that requires us to understand scattering at finite energy: the linear response conductance as a function of temperature. We compare it to Costi et al.'s NRG results [7] and find excellent agreement. This is important as it indicates we have an essentially correct description of the low energy scattering states.

Computing the linear response conductance at finite $\mathrm{T}$ is a complicated matter. We now have to compute the scattering phases of the glued charge and spin excitations in the presence of a 'thermalized ground state'; that is we must compute dressed scattering matrices. This ground state is no longer composed of merely real $\mathrm{k}$ states and $\lambda$ states of bound spin and charge as it was at $T=0$. 
Rather all the possible solutions of the Bethe ansatz equations of the model make an appearance.

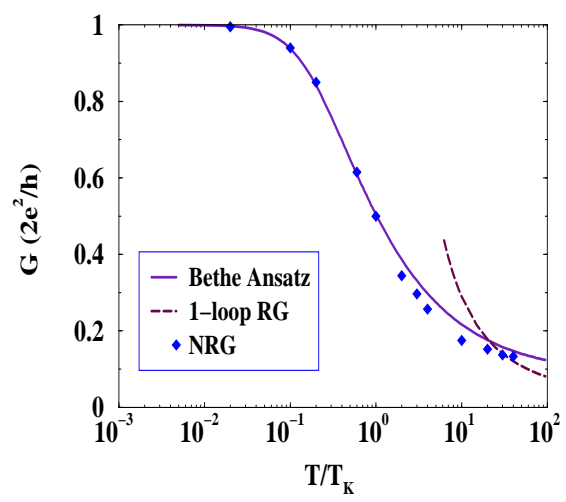

FIG. 2. A plot of the scaling curve for the conductance at the symmetric point as a function of $T / T_{k} . T_{k}$ is as defined in Costi et al.'s work and as such there are no free parameters.

However it is possible to derive equations governing the impurity densities $\rho_{\text {imp }}$ and $\sigma_{\text {imp }}$ in the presence of the complicated ground state and to solve numerically the corresponding set of coupled integral equations [12]. With these in hand, the linear response conductance is given by

$$
G(T)=\int_{-\infty}^{\infty} d \epsilon_{e l}\left(-\partial_{\epsilon_{e l}} f\left(\epsilon_{e l}\right)\right)\left|T\left(\epsilon_{e l}\right)\right|^{2},
$$

where $f$ is the Fermi distribution and $\left|T\left(\epsilon_{e l}\right)\right|^{2}=$ $\sin ^{2}\left(\frac{1}{2} \delta_{e l}\left(\epsilon_{e l}, T\right)\right)$ is the dressed scattering amplitude. A plot of the result and a comparison with the NRG results of [7] are given in Figure 2. For temperatures up to $T_{k}$, the regime where in principle an NRG computation should be of greatest accuracy, we find excellent agreement.

Because of the Fermi liquid nature of the problem when $T \ll T_{k}$, we know the functional form of the conductance is

$$
G\left(T / T_{k}\right)=1-c T^{2} / T_{k}^{2}+\cdots .
$$

Costi et al. [7], based upon results borrowed from [14,15], computed $c=\pi^{4} / 16=6.088$. We find from a fit of our curve, $c=6.05 \pm .1$. We have arrived at this value by fitting the plot in the region $T / T_{k}<.1$. The error is systematic in nature, arising from the arbitrary nature of deciding the region over which to fit.

We also compare our results in Fig. 2. with [17]. It would appear that the logarithmic dependence 17 characteristic of weak coupling and arising from a one-loop RG, should only be expected to become qualitatively descriptive for values of $T / T_{k}$ in excess of about 20 .

We have recently computed the finite temperature linear response conductance at the symmetric point in finite applied field. We again find reasonable agreement with [7].
Non-Equilibrium $(T=0, H \neq 0)$ : As we have successfully reproduced numerics on the finite temperature linear response conductance, we have some confidence that we understand scattering away from the Fermi surface. As such we can explore non-equilibrium conductances which depend upon the same information. Although there are issues relating on how to view the nonequilibrium system in the one-lead picture, these can be successfully handled [12]. We essentially adopt the Landauer-Büttiker approach taken in [16]: we employ the in-equilibrium scattering matrices; the sole role the bias plays is to set the particle distribution in each lead.

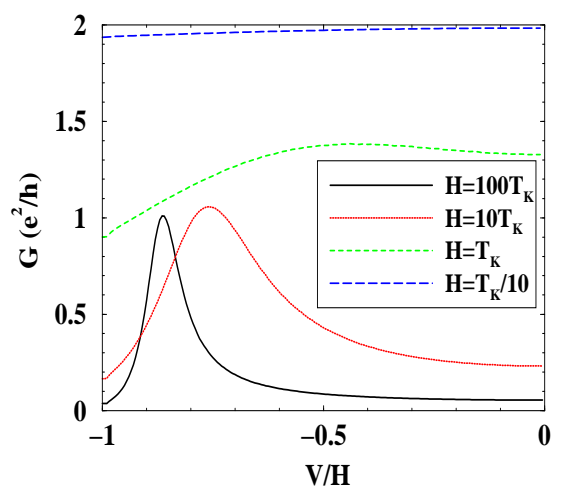

FIG. 3. A plot of the differential conductance in a magnetic field at the symmetric point with $U=8 \pi \Gamma$.

The current between the two leads, lead one at zero bias and lead two at bias $V<0$ is given by

$$
J(V)=\frac{e}{h} \int_{e V}^{0} d \epsilon\left(\left|T_{\uparrow}^{1 \rightarrow 2}(\epsilon)\right|^{2}+\left|T_{\downarrow}^{1 \rightarrow 2}(\epsilon)\right|^{2}\right),
$$

In Figure 3 we plot the differential conductance, $G=$ $-\partial_{V} J$, in the presence of a magnetic field. Our results are in rough accordance with [5] - we find that for fields, $H>T_{k}$, the zero-bias, zero field differential conductance peak divides in two, one peak for each spin species. Roughly speaking, the origin of the split in the differential conductance arises from a similar bifurcation in the impurity density of states. The spectral weight of the Kondo resonance present at zero energy when $H=0$ divides into two resonances near $V \sim \pm H$, again one associated with each spin species. However unlike [5] we find that the peak does not occur exactly at $|V|=H$ - in fact, the approach to this value is logarithmic in $H$ [12]. This discrepancy is unsurprising given the perturbative nature of the computation in [5].

In the large field limit, $H \gg T_{k}$, our analytical control allows us to employ a Wiener-Hopf analysis [12] to analyze the properties of the peak in the differential conductance. With $a=\log \left(H \sqrt{\pi e} / 2^{3 / 2} T_{k}\right) / \pi$, a peak maximum occurs at a bias, $e V_{\max }=-H\left(1-\cot ^{-1}(a) /(2 \pi)\right)$. The corresponding width of the peak is given by $e \Delta V=$ $H /(2 \pi)\left(\cot ^{-1}(a-1 / 2)-\cot ^{-1}(a+1 / 2)\right)$, while the height of peak is, $G_{\max }=e^{2} / h\left(3 / 2-a / \sqrt{4 a^{2}+1}\right)$. 
To conclude, we have shown that the integrability of the Anderson model can be exploited to compute transport quantities for quantum dots. The computation is more difficult than in the quantum Hall tunneling problem [16], and requires an ansatz to describe scattering away from the Fermi surface. The accuracy obtained in reproducing known numerical results [7] is remarkable, and suggests that the method could be a successful way of handling non-equilibrium transport in general integrable systems. In future work, we will further exploit this technique to compute the DC shot noise at zero temperature in quantum dot systems [18].

R.K. has been supported by NSERC, the NSF through grant number DMR-9802813 and through the Waterman Award under grant number DMR-9528578, and the University of Virginia Physics Department. H.S. has been supported by the Packard Foundation, the NYI Program, and the DOE (H.S.). H.S. also acknowledges hospitality and support from the LPTHE (Jussieu) and LPTMS (Orsay). AWWL has been supported by the NSF through grant DMR-00-75064.

[1] D. Goldhaber-Gordon, J. Göres, M. Kastner, H. Shtrikman, D. Mahalu, and U. Meirav, cond-mat/9807233; D. Goldhaber-Gordon, H. Shtrikman, D. Mahalu, D. Abusch-Magder, U. Meirav, and M. Kastner, Nature 391 (1998) 156.

[2] S. Cronenwett, T. Oosterkamp, and L. Kouwenhoven, cond-mat/9804211.

[3] D.C. Ralph, R.A. Buhrman, Phys. Rev. Lett. 72 (1994) 3401.

[4] T. Ng and P. Lee, Phys. Rev. Lett. 61 (1988) 1768.

[5] Y. Meir, N. Wingreen, and P. Lee, Phys. Rev. Lett. 70 (1993) 2601; N. Wingreen and Y. Meir, Phys. Rev. B 49 (1994) 11040; N. Sivan and N. Wingreen, Phys. Rev. B 54 (1996) 11622.

[6] S. Hershfield, J. Davies, and J. Wilkins, Phys. Rev. B 46 (1992) 7046; M. Hettler, J. Kroha, and S. Hershfield, cond-mat/9707209.

[7] T. Costi, A. Hewson, and V. Zlatic, Journal of Physics: Cond. Mat. 6 (1994) 2519; T. Costi, cond-mat/0004302.

[8] J. Moore and X. Wen, cond-mat/9911068.

[9] D. Langreth, Phys. Rev. 150 (1966) 516.

[10] N. Kawakami and A. Okiji, Phys. Lett. A 86 (1982) 483; ibid. J. Phys. Soc. Japan 51 (1982) 1143; ibid Solid St. Commun. 43 (1982) 365; Okiji, A. and Kawakami, N., J. Phys. Soc. Japan 51 (1982) 3192.

[11] P. Wiegmann, V. Filyov, and A. Tsvelick, Soviet Phys. JETP Lett. 35 (1982) 77; P. Wiegmann and A. Tsvelick, Soviet Phys. JETP Lett. 35 (1982) 100; ibid., J. Phys. C 16 (1982) 2281; A. Tsvelick and P. Wiegmann, Phys. Lett. A 89 (1982) 368; ibid., J. Phys. C 16 (1983) 2281; V. Filyov, A. Tsvelick, and P. Wiegmann, Phys. Lett. A 89 (1982) 157.
[12] R. M. Konik, H. Saleur, and A. W. W. Ludwig, in preparation.

[13] N. Andrei, Phys. Lett. A. 87 (1982) 299.

[14] J. Nozières, J. Low Temp. Phys. 17 (1974) 31.

[15] K. Yamada, Prog. Theo. Phys. 53 (1975) 970.

[16] P. Fendley, A.W.W. Ludwig, and H. Saleur, Phys. Rev. B52 (1995) 8934.

[17] A. Kaminski, Y. Nazarov, and L. Glazman, condmat/0003353.

[18] R. M. Konik, H. Saleur, and A. W. W. Ludwig, in preparation. 